\title{
Sabiduría de un autodidacta sobre la educación
}

\author{
Wisdom from a self-taught individual on education
}

\author{
MIGUEL ÁNGEL VILCHES SILVA \\ Profesor de Historia y Geografía (UCSH) \\ Magíster en Educación Basada en Competencias (UTALCA) \\ Coordinador de Desarrollo Curricular \\ Universidad Autónoma de Chile. Sede Talca, Chile. \\ miguel.vilches@uautonoma.cl
}

\section{RESUMEN}

Educar a las nuevas generaciones implica una tensión con las generaciones predecesoras, pues las últimas dirigen el proceso formativo de los sujetos que se están integrando. Por otra parte, los modelos educativos son el reflejo de la sociedad que ha optado por él, a fin de sustentar -teóricamente y con procedimientos- las acciones (u omisiones) en el arte de formar a los alumnos. Por ello, este trabajo desarrolla la perspectiva educativa de Roberto Hernández Cornejo existente en la obra "Los exámenes y bachillerato. Algunos aspectos sobre la crisis de nuestra enseñanza" (1939). Este autor, desde su formación intelectual y desempeño profesional autodidacta, plantea críticas a elementos estructurales del curriculum en la educación, contrastándola con experiencias educativas distantes y autores que esgrimían la necesidad de modernizar la forma de educar y evaluar a los estudiantes. En cuanto al desarrollo del texto, se inicia con una contextualización histórica y pedagógica al autor, para, después, delimitar su crítica y propuesta certera frente a la situación educativa que analiza.

Palabras clave: educación, currículum, paradigma, evaluación, autodidacta, determinismo social, naturalismo. 


\section{ABSTRACT}

Educating new generations means a tension with the predecessors, the ones that direct the process to wards the individuals who are being integrated. The educational models are a reflection of the society that has chosen it in order to support theoretically and with procedures the actions (or omissions) in the art of training new members. The study develops the educational perspective of Roberto Hernández Cornejo existing in the work "The exams and diploma. Some aspects about our teaching crisis" (1939). The autor from his intellectual training and self-educated professional performance, brings up criticisms to the structural elements of the curriculum in education, contrasting it with distant educational experiences and authors who made an argument of the need to bring up to date the way to educate and assess students. Regarding the development of the text, it begins with a historical and pedagogical contextualization of the author, then defines his criticism and accurate proposal regarding the educational situation that he is analyzing.

Key words: Education, curriculum, paradigm, evaluation, self-taught, social determinism, naturalism.

\section{INTRODUCCIÓN}

Roberto Hernández Cornejo (1877-1966) fue un periodista, historiador y bibliófilo, que nació en Melipilla en 1877, durante el gobierno de Aníbal Pinto (18761881). Es el segundo de los 16 hijos que tuvo el matrimonio de Natalia Cornejo y Wenceslao Hernández.

Don Roberto recibió una educación formal hasta segundo grado de educación primaria -la instrucción obligatoria se implementó desde 1920. En los años posteriores, solo tuvo una formación autodidacta. La profundidad de sus conocimientos lo llevó al grado de erudición histórica.

Con esas herramientas, desde el año 1894, cuando Hernández Cornejo tenía 17 años, inició su trabajo en distintos periódicos (La Constitución, El Deber y La Unión) sobre historias poco conocidas en el ámbito regional y local, llegando a constituirse a sí mismo en erudito del pasado, mediante el estudio y publicación: "Yo no trabajé sino en dos cosas que en mí ejercieron desde muy niño la franca 
e irresistible atracción (...) el periodismo y las funciones propias del bibliotecario" (Hernández, 1958, p. 4). En 1902, continuó su labor en la capital en el diario El Chileno, desempeñándose como secretario de redacción y realizando campañas editoriales y reclamaciones de justicia. Sin embargo, tras el terremoto de 1906 en Valparaíso, con 29 años, Roberto llegó a la ciudad puerto, asumiendo la dirección del periódico El Chileno. Desde 1915, lo vemos como redactor de crónicas históricas en La Unión, de Valparaíso.

Entre 1917 y 1953, fue el conservador y director de la biblioteca pública Santiago Severín. Ese contexto, será el nicho de mayor auge de sus estudios e investigaciones, artículos y publicaciones: editó 28 libros, principalmente de historia nacional y local, sin dejar de tratar el ámbito educativo, pues la actualidad de ese tema lo motivó a investigar y hacer algunas reflexiones sobre el asunto. Por su destacada labor, Hernández Cornejo también obtuvo el reconocimiento de la Real Académica de la Historia de Madrid (1921) y de la Academia Chilena de la Lengua (1940). Don Roberto falleció en el puerto, el 11 de enero de 1966, a la edad de 89 años.

\section{DESARROLLO}

Roberto Hernández, en su obra Los exámenes y bachillerato. Algunos aspectos sobre la crisis de nuestra enseñanza (1939), compila distintos artículos publicados en el diario La Unión, bajo ese nombre, durante los meses de febrero y marzo de ese año, como consecuencia de la reprobación casi "total" del examen de bachillerato, condición de la cual se exceptuó solo un alumno de un curso.

El libro, en su primera parte, presenta una serie de textos que aluden a: la utilidad de los exámenes, consecuencia de estos, validez de la forma de evaluar, misión del Estado en la enseñanza, libertad de enseñanza y definición sobre qué es aprender, junto a una propuesta de supresión de los exámenes por ser estos en sí mismos aleatorios. La segunda parte del texto incluye distintos temas educativos, demostrando que las condiciones educativas ya venían discutiéndose sin resultados efectivos a la fecha. Al respecto, se abordan las condiciones económicas y los malos resultados en la educación, cantidad de horas, estado de la educación (primaria y secundaria) y los desafíos de mejoramiento en las escuelas, específicamente, en la educación secundaria y su bachillerato, entre otros temas relacionados con la enseñanza. 
Ahora bien, ¿cuáles fueron las motivaciones de Hernández Cornejo sobre este tema? El escrito nos interesa por su actualidad y vigencia. Por eso, se hará ver el contexto de las inquietudes de Roberto Hernández $(\mathrm{RH})$ y, después, se integrarán los elementos curriculares observados en la obra aludida con dos exponentes teóricos: Spencer y Dewey.

En ese sentido, la década de 1930 fue testigo privilegiado de vicisitudes que modificaron permanentemente la forma en la cual el hombre buscó responder a preguntas básica sobre su naturaleza, el bien común y la forma en la que se relaciona con sus pares. Ese período fue producto del caos de las democracias liberales y el colapso económico del Jueves Negro (1929), hitos que demarcarán distintas iniciativas entre las cuales se encuentra el: New Deal de Franklin Delano Roosevelt; los intentos fascistas de Mussolini en Italia o de Hitler en Alemania; 0 , el fortalecimiento del proyecto económico marxista de la URSS (Abbagnano, 1992).

En el cambio de rumbo político, económico, social y educativo, se refleja la tesis de Tomás Kuhn, quien propone que "los paradigmas son realizaciones científicas que en primer lugar carecen de precedentes como para atraer a un grupo duradero de partidarios, pero que son lo suficientemente incompatibles como para dejar problemas a resolver" (p. 41), por lo mismo, -referente con acuerdos, desacuerdos y exponentes- es que constantemente existe un cambio de paradigmas en distintas crisis que experimenta la sociedad como conjunto.

Los pilares educativos de la época eran, por un lado, el alicaído método francés y, por otro, el germánico, cuya propuesta centralista y supervisada estaba cimentada como ciencia positiva decimonónica, que estima la construcción de habilidades cuantitativas mediante la instrucción a los alumnos, quienes como tabula rasa debían moralizarse, siendo funcionales al sistema para que suscite curiosidad, interés y búsqueda. Asimismo, como consecuencia de la Primera Guerra Mundial y de la crisis económica mundial, especialmente en Europa, se generó el espacio (crisis) para que nuevos modelos se superpusieran, siendo el momento del modelo norteamericano (Escuela Nueva) como exponente pedagógico principal. Además, autores como Cousinet (francés), Piaget (suizo), Montessori (italiana), Ferreire (suizo), Kerchenteiner (alemán), Spencer (inglés) y Dewey (norteamericano) cobraron relevancia.

El Estado chileno en la década de 1930 heredó un fuerte tinte social. La constitución de 1925, estableció en el artículo № 11 que: "es deber del Estado 
velar por la salud pública y el bienestar higiénico del país" (BCN, 1925, p. 8). Esta garantía constitucional fue la base del Estado docente, el cual específicamente atendería y velaría por las condiciones educativas de la República.

Al respecto, la educación primaria desde 1920 se asume como obligatoria, siendo todo el sistema vigilado por una superintendencia que desempeñaba la Universidad de Chile. Pero, la preocupación por los distintos niveles educativos tuvo distinciones, considerando, por ejemplo, el nivel, el gasto universitario era más de 25 veces superior al gasto por alumno en educación básica (Soto, 2004).

De esa manera, el gasto público debió esperar hasta 1960 para reformar la educación primaria, pudiendo así superar unas de las deudas históricas, que era más del 43,9\% de analfabetismo de la población (Silva, 2015). Por otro lado, sobre la educación universitaria, el país alcanzaba un $1.06 \%$ de cobertura, mientras que Estados Unidos llega al $4.7 \%$, nivel que será alcanzado solo 20 años más tarde (UDD, 2008).

En ese contexto educativo, Chile estaba sufriendo un punto de inflexión particular: la toma del poder por los sectores medios, que promovieron el desarrollo de valores laicos en la educación como herramienta de desarrollo social.

Si bien la finalidad de la enseñanza es formar habilidades en los hombres para que se desarrollen a sí mismos en una cultura desinteresada (Gómez, 1928, p. 33), entonces será deber del Estado proporcionar las condiciones básicas, respetando la libertad de cátedra y el espíritu del profesorado (Gómez, 1928). Los radicales, con su lema "gobernar es educar", tratarán de transmitir una nueva conciencia moral, quedando en el recuerdo colectivo el ideario político de sus gobiernos, que se enfrentaron a las consecuencias de la crisis económica, al auge de gobiernos totalitarios y a la oposición del sector tradicional católico sobre los fines de la educación (Mardones, Fierro y Salas, 2016).

Los años 30 demuestran la lucha de dos principios: el derecho a la educación y la libertad de enseñanza (Peña, 2015). El primero es entendido como un derecho social proclamado desde fines de la revolución francesa para poder mantener la sociedad; y, el segundo, respecto del currículum, será determinar el: ¿qué?, ¿cómo? y ¿cuándo? enseñar y evaluar.

Como lineamiento pedagógico de la época, en Chile se desarrolló la ideología liberal educativa con educadores como Letelier, Matte, Gallo, cuyo apremio 
fue dar respuesta al problema nacional con diferentes hitos como: la Asamblea Pedagógica (1926), el Ministerio de Educación Pública (1927), el Congreso Pedagógico y la reforma educativa (1928); hasta que, llegó el gobierno de Pedro Aguirre Cerda, producto del Frente Popular y como representante de sectores laicos (1935).

Económicamente, Chile optó por la Industrialización por Sustitución de Importaciones (ISI) para la cual era necesario formar las habilidades en armonía con los intereses de la sociedad (educación chilena para los chilenos). Fue así, que se propuso expresamente la continuidad de los niveles educativos hasta la universidad, cuyo principal sostenedor será el Estado. Escuela Nueva fue el nombre de este movimiento reformista (Reyes, 2010).

La educación primaria y secundaria en la época vieron surgir diferentes referentes como el periódico Nuevos Rumbos y Boletín Nacional (1926-1928), la creación de liceos experimentales (Manuel de Salas, 1933). Sin embargo, el ímpetu de reforma se contuvo en la educación primaria y secundaria, pues la educación universitaria se exceptuó, siendo el movimiento liderado por la Universidad Católica de Chile, que desde el año 1926 organizó una serie de conferencias pedagógicas, cuyos objetivos era mantener el ethos católico universitario, frente al nuevo modelo educativo. No se rechazó explícitamente a los exponentes de la Escuela Nueva, pero sí sus fines mecanicistas y naturalistas frente a la doctrina católica romana propuesta por Pio XI en su encíclica Divini Illus Magistrari (Caisedo, 2011, p. 7-10).

Adentrándose desde el contexto, la vertiente pedagógica de Roberto Hernández demuestra coincidencia con dos propuestas: una de Spencer y la otra de Dewey.

Herbart Spencer (1820-1903) fue un amateur learning, nacido en Reino Unido, que precozmente demostraba aptitudes para el aprendizaje a través de la experiencia. El autor contemporáneo a Darwin, apoyó el optimismo en el ser humano, quien está determinado (Holmes, 2002) a aprender para poder así adaptarse y responder a los desafíos que la sociedad impone (educación evolucionista). Se exalta el valor de la motivación intrínseca como herramienta didáctica de aprendizaje, que no debe estar determinado centralizadamente por el Estado; e incluso, llevado al extremo, la educación formal y su currículum sería algo innecesario y coercitivo.

Por otro lado, se encuentra la propuesta de John Dewey (1859-1952), un norteamericano que propone un sistema pedagógico racionalista, cuya finalidad 
en la educación es entregar a los estudiantes representaciones que sirvan para desarrollar conceptos lógicos y claros, a fin de promover la democracia mediante la educación, para así "promover en los individuos un interés personal en las relaciones y control social y los hábitos espirituales que produzcan cambios sin generar desorden" (Dewey, 1925, p. 103). En ese contexto, será importante promover el learning by doing (aprender haciendo), en la cual el sustrato básico es que todo el conocimiento debe cimentarse en la experiencia, para así fortalecer la educación intelectual, insertando trabajos manuales y desarrollo manual (educación naturalista).

Pedagógicamente, desde la sapiencia que entrega el ser autodidacta, ¿qué críticas curriculares plantea RH? En consistencia con los autores destacados durante la coyuntura que surge como dilema de la utilidad del examen de bachiller se observa:

Nadie ha echado la culpa al verdadero culpable, el examen mismo (...) todos sabemos que si existe algo aleatorio; algo que no prueba nada es el examen (...) existe todo tipo de alumnos que sabiendo todos menos un dato insignificante se lo preguntaron y salió mal, otros en cambio sabían una sola cosa y salieron bien. Un profesor que se ocupa de su clase llena una ficha de cada uno de sus alumnos que los hace trabajar y tienen referencias no necesita para dar el paso al curso superior de esa prueba final (Hernández, 1938, p. 6 y 41).

Miguel Santo Guerra (2003), especialista en didáctica, posee una frase aclaratoria al dilema: "Dime cómo evalúas y te diré qué tipo de profesional y persona eres". Entonces, se entiende que el juicio del autor no es una crítica somera, sino más bien profunda, que surge de su propia experiencia e intuición: ¿Qué tipo de educación se está impartiendo?, ¿Qué valores se enseñan?, ¿Cuáles son las consecuencias de utilizar la evaluación en estas condiciones para todo el quehacer curricular? A fin de responder a las interrogantes desde la fuente aludida curricularmente se concibe:

1. Sobre el conocimiento: $\mathrm{RH}$, sin validaciones, plantea -frente a resabios del positivismo educativo y la mentalidad instrumental de la época- la fascinación por el saber desde la experiencia autodidacta de alguien que contempla lo que sabe, lo que hace y se da cuenta de cuánto más podría realizar. ¿Por qué? En ese entonces, los estudiantes aprenden, pese a las prácticas pedagógicas descontextualizadas. 
Importante es hacer notar que, el autor no propone aniquilar la educación formal (propuesta de Spencer), sino más bien reorientar el foco de atención en la formación de pensadores creativos, con autocontrol y hábitos que cimientan el aprendizaje (Naturalismo Dewey), sabiendo controlar la impulsividad mediante la conciencia de su propio pensamiento:

La mayor parte de los hombres en quienes vive la afición a los estudios, se mantienen por estimulo noble de investigar algo nuevo y ese estímulo se apaga 0 amortigua cuando se le hace creer al alumno que todo está resulto, ya averiguado y sabido (Hernández, 1938, p. 8).

Llama la atención cómo intuitivamente Hernández, se adelantó incluso a un fenómeno social desconocido, que es la desmotivación educativa. El problema educativo en el primer cuarto del XX, fue la cobertura educativa para luchar contra el analfabetismo en los niveles inferiores del sistema educativo, sin trastocar la estructura metodológica "litúrgica" universitaria.

$\mathrm{RH}$ va más allá en su propuesta -evolucionista según Spencer-, es decir, desde una condición innata en el ser humano por desarrollar herramientas para así poder responder e innovar soluciones más allá de repetir algoritmos. Desde el autor, se entiende que aprender no es un trauma, sino una fascinación cercana al conocimiento y experiencias previas de los estudiantes, aprovechando la oportunidad de provocar sus emociones, atención y preparándolo para responder al cambio -coincide con las tesis de Spencer y Dewey- que socialmente experimentará durante su desarrollo como ser social, superando con esto el abismo entre la experiencia y las diferentes materias que constituyen el programa escolar: "El saber memorizado sin ningún fin aprovechable, carece de sentido y no es más que un adorno de la mente" (Hernández, 1938, p. 26).

2. Sobre el currículum: $\mathrm{RH}$ propone -en concordancia con Spencer (evolucionismo social) y Dewey (educación naturalista)- que sin la experiencia, cualquier proceso de aprendizaje se inhibe, quedando solo en la esfera de la memorización. En este punto, la educación como instrucción será una herramienta innecesariamente coercitiva, debido a la potencialidad innata de los individuos, quienes están capacitados para responder a las exigencias del medio. Por lo mismo, habrá que rescatar un lenguaje claro y cercano a los estudiantes para que así la pedagogía deje de ser un ejercicio antagónico a la juventud y responda a las tendencias de desarrollo de los estudiantes, frente a los desafíos de la sociedad mayor, en la cual se están insertando: 
Será necesario dotar al educando de las nociones de ciencias aplicadas a su profesión, arte u oficio, pero no existe necesidad alguna de embutirle en el cerebro quepa o no un extracto de todo el saber humano que se complejizan aún más siendo incluso una pérdida de tiempo (Hernández, 1958, p. 41).

3. ¿Qué y cómo se enseña? Según la tesis de Durkheim, en la Educación Social, la sociedad transmite a las nuevas generaciones la conciencia moral, adquiriendo con esto los valores, principios y disposiciones en la vida y las costumbres, a fin de decidir asertivamente como proceder. Es decir, en la experiencia universitaria aludida, los estudiantes debían rendir 45 exámenes antes del bachillerato. ¿Qué hacer con ellos? RH es claro "valdría la pena suprimirlos", puesto que "no hay ninguna necesidad de imponer al aspirantado una tarea demasiado gravosa ya que se corre el peligro de abrir ancho y expedito a la ignorancia atrevida e impaciente" (Hernández, 1938, p. 38).

La recolección de información para poder tomar decisiones (evaluación) es el detonante y evidencia todo el quehacer educativo, razón por la cual se puede dar oportunidad para que el proceso de aprendizaje fluya constantemente para así poder rescatar experiencias como:

Los antiguos comerciantes enseñaban de una forma práctica, haciéndose acompañar de los discípulos en su labor. Esa escuela práctica era la única conocida y es la misma que tienen en el extranjero, al paso que nosotros nos obstinamos en un sistema de enseñanza empírico que no toma en cuenta las necesidades del país y que ha repercutido aparentemente en la economía nacional (Hernández, 1938, p. 25).

4. Docentes y estudiantes: queda de manifiesto que, las decisiones y estrategias metodológicas que los docentes toman al momento de desarrollar el currículum es constituyente de un factor pedagógico sobre las formas de interacción, estrategias metodológicas y formas de evaluación:

¿Qué pensar de exámenes que hacen incidir sobre materias, con el recargo de los programas que el profesor no ha tenido nunca tiempo de pasar en clases"? hay preguntas que son una verdadera adivinanza (Hernández, 1938, p.14).

Su propuesta se acerca a la perspectiva paidocéntrica, centrada en el aprendizaje, conocimientos previos y prácticos como una necesidad, razón por lo cual el docente debe facilitar las experiencias para que sus estudiantes resuelvan 
problemas o casos -coincidencia con Dewey y Spencer. Es decir, la determinación de objetivos instruccionales solo genera consecuencias insatisfactorias, pudiendo resaltar la falta de atractivo propio de la disciplina y ambigüedad de las calificaciones, condiciones que, en cambio, deberían estimular la motivación para alcanzar altos niveles de aprendizaje, dando la posibilidad de un aprendizaje sin límites, sin dejar de lado la libertad de enseñanza (Hernández, 1938) y la responsabilidad de los padres o apoderados en la enseñanza de sus pupilos (Hernández, 1938).

Por otro lado, -complementando la idea-, al considerar al estudiante como tabula rasa, homogenizando sus expresiones naturales a procedimientos estándares solo produce un abismo con la realidad natural. Las ciencias exactas $y$, lentamente, las ciencias sociales, han demostrado que la riqueza se encuentra en la heterogeneidad de los participantes, puesto que, desde sus características propias, están enfrentados a responder los problemas que la complejidad social produce:

¿Cómo no tener presente que todo estudiante no puede poseer igual facilidad de expresión, tranquilidad, dominio de los nervios y memoria? Se corre el peligro de generar una indigestión del espíritu (Hernández, 1938, p. 13).

¿Qué consecuencias produce una educación de estas características? El quehacer pedagógico comúnmente depende del dominio del estado del arte y la habilidad didáctica del docente. $\mathrm{RH}$, sin ser pedagogo, se acerca intuitivamente a las causas del dilema, ya que desde su experiencia autodidacta se acercó a la necesidad de dar un repaso de la educación. La educación debe ser una brújula, formar individuos y debe crecer el espíritu social, integrando experiencias e intereses: "Educar en esta forma a jóvenes en ignorancia, falta de concepto lógico, incoherente y tontificación" (Hernández, 1938, p. 38).

\section{CONCLUSIÓN}

En general, el contexto educativo con aciertos y errores, provoca siempre la reacción en los sujetos que lo constituyen. Estos, por ser seres sociales se encuentran condenados a reproducir o modificar las pautas (paradigmas) a seguir en una evolución cíclica que busca constantes respuesta a vicisitudes contingentes. Roberto Hernández no es la excepción, ya que no fue indiferente a elementos corrientes en la época -la reprobación- desde los cuales levantó su crítica lúcida. 
El proceder y saber docto institucional merece la oportunidad y ser revisado según la "sapiencia" de alguien que sorteó las barreras comunes a un perfeccionamiento personal y profesional. Desde una perspectiva, improbable a una mera imposición oficial del currículum, Roberto Hernández fue capaz de nutrir una propuesta de educación laica y en libertad de enseñanza muy coincidente con los postulados de Herbart Spencer y John Dewey. La educación evolucionista, exalta la respuesta del individuo frente a la presión social, siendo los más aptos quienes responden. El autor, no solo responde, sino que excede el horizonte común de desarrollo de sus contemporáneos. ¿Qué produjo esta situación? Desde la tesis de Dewey, se puede desvelar una respuesta simple y concreta: el aprender haciendo, más allá de solo proceder, pues genera reflexión en los sujetos motivando su actuar, dando sentido al quehacer diferentes áreas incluso insospechadas.

La experiencia autodidacta, entendida como "aquel que se instruye por sí mismo" evidencia en RH esta cualidad perfeccionada con disciplina y curiosidad desde la primera infancia hasta su deceso. Las dificultades, no fueron un obstáculo, más bien desde la experiencia y el rigor, fue advirtiendo aquello que lo motivaba: el conocimiento como herramienta en temáticas históricas y sociales poco investigadas.

¿Qué legitimad tendría un sistema educativo abiertamente contrario a su experiencia de aprendizaje? Ninguna, ya que considerando el conocimiento de libre acceso, y la educación como una herramienta de desarrollo, el currículum oficial trae como consecuencia adormecer el potencial de los estudiantes. Entonces, únicamente docentes y aprendices motivados podrán acceder a la pasión por su labor, dominando sus habilidades y respondiendo efectivamente a razones que en su interior tendrán sentido, pero que, a la vez, beneficiarán también a su grupo social. 


\section{REFERENCIAS BIBLIOGRÁFICAS}

ABBAgnanO, N. (1992). Historia de la Pedagogía. Fondo de Cultura Económica. p. 502.

BCN (1925). Constitución de Chile. Biblioteca Congreso Nacional p. 46. Disponible en: https://www.bcn.cl/historiapolitica/constituciones/detalle_constitucio n?handle=10221.1/17659. Accedido el: 17-10-18.

CAICEDO, J. (2011). "Pensamiento pedagógico en Chile en el siglo XX: desde la escuela nueva al constructivismo". Revista Teoría e Práctica da Educação, ํㅜ 14. pp. 7-20. Disponible en: http://periodicos.uem.br/ojs/ index.php/TeorPratEduc/article/view/16148. Accedido el: 17-10-18.

DEWEY, J. (1925). El niño y el programa escolar. Disponible en: http://repositorio. educacion.gov.ar:8080/dspace/handle/123456789/107975. Accedido el: 17-10-18.

DURKHEIM, E. (1976). La educación como socialización. Ediciones Sígueme. Salamanca. p. 274.

GÓMEZ, L. (1928). Revista de educación primaria. pp. 30-33, 36-42. En Reyes, L. (2010) "Movimiento educacional, crisis educativa y reforma de 1928". Revista Docencia $n^{\circ}$ 40. Programa Interdisciplinario de Investigaciones en Educación. pp. 40-49. Disponible en: http://piie.cl/biblioteca/ publicaciones/articulos/A-2010-015.pdf. Accedido el: 17-10-18.

HERNÁNDEZ, R. (1938). Los exámenes y el bachillerato. Algunos aspectos sobre la crisis de nuestra enseñanza. "La Unión" de Valparaíso. Imperial. p. 97.

HERNÁNDEZ, R. (1958). Vistazo periodístico a los ochenta años. Imprenta Victoria, Valparaíso. p. 122.

HOLMES, B. (2002). Herbert Spencer. Unesco, vol. 24 pp. 533-554. Disponible en: www.ibe.unesco.org/sites/default/files/spencere.pdf. Accedido el: 17-10-18.

KUNH, T. (2004). La estructura de las revoluciones cientificas. Fondo de Cultura Económica. México. p. 318. 
MARDONES, R., FIERRO, C. Y SALAS, G. (2016). "Cuestión social en Chile: Discursos sociales y sus referencias en los saberes 'PSI"' (1880 -1930). Revista Historia de la Psicología № 37. pp. 8-15. Disponible en: https:// www.researchgate.net/publication/299447169_Cuestion_social_ en_Chile_discursos_sociales_y_sus_referencias_a_los_saberes_ psi_1880-1930_The_social_question_in_Chile_Social_discourses_ and_their_references_to_Psy-knowledge_1880-1930. Accedido el: 17-10-18.

PEÑA, C. (2015). Derecho a la educación y libertad de enseñanza. Centro de Estudios Públicos. № 143. Disponible en: https://www.cepchile.cl/ cep/site/artic/20161004/asocfile/20161004122606/rev143_cpena.pdf. Accedido el: 17-10-18.

REYES, L. (2010). Movimiento educacional, crisis educativa y reforma de 1928. Revista Docencia $N^{\circ} 40$. Programa Interdisciplinario de Investigaciones en Educación. pp. 40-49. Disponible en: http://piie.cl/biblioteca/ publicaciones/articulos/A-2010-015.pdf. Accedido el: 17-10-18.

SANTOS, M. (2003). "Dime cómo evalúas y te diré que tipo de profesional y de personas eres". Revista Enfoques Educacionales № 5. pp. 69-80. Disponible en: http://www.facso.uchile.cl/publicaciones/enfoques/07/ Santos_DimeComoEvaluas.pdf. Accedido el: 17-10-18.

SILVA, B. (2015). Historia de la educación chilena, Tomo I. Instalación, auge y crisis de la reforma alemana. Ediciones Universidad Tecnológica Metropolitana. Santiago, Chile.

SOTO, M. (2004). Políticas educacionales en Chile durante el siglo XX. Facso. Mayo $\mathrm{N}^{\circ}$ 10. Disponible en: www.facso.uchile.cl/publicaciones/mad/10/ paper04.pdf. Accedido el: 17-10-18.

UDD (2015). Sin educación tampoco hay mejor distribución del ingreso. Facultad de Negocios UDD. Disponible en: http://negocios.udd.cl/files/2015/08/ Presentaci\%C3\%B3n-CLV.pdf. Accedido el: 17-10-18. 